LLAMAS, F. -1984- Flora y Vegetación de la Maragatería (León). Inst. Fray Bernardino de Sahagún. Excma. Dip. Prov. León.

LOSA, T.M. -1941- Plantas de los alrededores de Riaño (León). Anales Jard. Bot. Madrid, 2:172-188.

LOSA, T.M. -1957- Catálogo de las plantas que se encuentran en los montes palentino-leoneses. Anales Inst. Bot. Cavanilles, 15:243-376.

LOSA, T.M. \& MONTSERRAT, P. -1951- Aportación al estudio de la Flora de los Montes Cantábricos. Anales Inst. Bot. Cavanilles, 10(2):423-510.

MERINO, P.B. -1905- Flora de Galicia. 3 vol. Tipografía Galaica. Santiago de Compostela.

MORALES VALVERDE, R. -1986- Taxonomía de los géneros Thymus (excluída la sección serpyllum) y Thymbra en la Península Ibérica. Ruizia, 3.

PUENTE GARCIA, E., PEREZ MORALES, C. \& LOPEZ PACHECO, M.J. -1984- Nuevos datos para la flora leonesa. Lazaroa, 6:297-299.

ROMERO, C. -1983- Flora y vegetación de la cuenca del río Luna (León). I.C.O.N.A. Monografías 29. Ministerio de Agricultura. Madrid.

TUTIN, P.G. \& al. -1964- Flora Europaea. I, Cambridge University Press.

WILLKOMM, M. \& LANGE, J.M.C. -1870/1880- Prodromus Florae Hispanicae. 3 vol. E. Schweizebart'sche Verlagsbuchhandlung. Stuttgart.

Dirección de los autores: Departamento de Biología Vegetal (Botánica). Facultad de Biología. Universidad de León.

\title{
CONTRIBUCIONES AL CONOCIMIENTO DE LA FLORA DEL NW DE MURCIA. II.
}

\author{
Caridad SELMA \& Oswaldo SOCORRO
}

RESUMEN: Se incluye en este trabajo las citas de 26 taxones del NW de la provincia de Murcia, de las que 24 de ellas resultan ser nuevas para la provincia.

SUMMARY: In this paper 26 taxa from NW of province Murcia are reported, 24 of these taxa are new records for this province.

Continuamos con esta nota la serie emprendida anteriormente Selma \& Socorro (1985). Con ellas queremos dar a conocer citas nuevas o criticas, o bien confirmar las ya existentes para la flora tanto del $\mathrm{NW}$ como a nivel provincial. Ello es el 
resultado de los trabajos que en la actualidad realizamos sobre

1 a flora y vegetación de la zona NW de Murcia.

Los pliegos testigos se encuentran incluidos en el Herbario

de la Universidad de Murcia, de los cuales damos el número de registro.

Alliaria petiolata (Bieb.) Cavara \& Grande

Moratalla, Sierra de Moratalla (WG6413), 1750 m, 7-VI-1981, no reg. 12475. Rara, como nemoral-nitrófila. Novedad provincial.

Anchusa arvensis (L.) Bieb. subsp. orientalis (L.) Nordh.

Cehegín, Casa del Cerrojo (XH0418), 600 m, 13-V-1984, no reg. 12478; Caravaca, Cortijo de la Hoya (WH9411), 800 m, 27-VI-1984, no reg. 12479. Poco frecuente, en campos de cultivos e inmediaciones a viviendas. Novedad provincial.

Androsace maxima L.

Caravaca, El Moral (WH7106), 1100 m, 21-IV-1984, no reg. 11394; Moratalla, Cortijo El Frontón (WH8628), 1200 m, 29-V-1982, no reg. 12477. En pastizales terofíticos de claros de espartales. Novedad provincial.

Carduncellus araneosus Boiss. \& Reuter

Moratalla, Sierra de la Muela (WH9135), 1200 m, 20-VI-1981, no reg. 12494; Caravaca, Sierra de Mojantes (WH8411), 1000 m, 11-VI-1982, no reg. 12496. Poco frecuente, en campos incultos. Hasta el momento solo ha sido señalada su presencia en la provincia, concretamente en Archivel, por Gandoger (1917:161).

Cerastium semidecantrum L.

Caravaca, Casas de Moya (WH8021), 1200 m, 9-VI-1984, no reg. 12490. Escasa, en pastizales terofíticos sobre suelos calizos. Novedad provincial.

Ceratocephala falcata (L.) Pers. var. barrelieri León Dufour

Moratalla, Sierra de la Muela (WH9139), 900 m, 28-IV-1984, na reg. 12500. Rara, en cultivos abandonados. Novedad provincial.

Dactylorhiza elata (Poiret) Soó subsp. sesquipedalis (Willd.) Soó

Moratalla, Sierra de la Muela (WH9136), 1300 m, 2O-VI-1981, no reg. 6780. Poco frecuente, en prados húmedos de Holoschoenetalia. Novedad provincial.

Euphorbia mariolensis Rouy

Caravaca, El Reventón (WH9421), 1300 m, 15-V-1982, nํ reg. 12489. Escasa, en laderas pedregoso calizas. Novedad provincial.

Euphorbia minuta Loscos \& Pardo

Caravaca, Sierra de Mojantes (WH8308), $1000 \mathrm{~m}, 22-\mathrm{V}-1982$, no reg. 12487: Moratalla, Casa de Cristo (WH9427), 950 m, 1-VI-1985, no reg. 12488. Poco frecuente, en romerales de laderas pedregoso calizas. Novedad provincial.

Epilobium parviflorum Schreber

Moratalla, Puerta de Moratalla, río Benamor (WH9030), $700 \mathrm{~m}$, 5-VII-1982, no reg. 12480. Poco frecuente, en márgenes de corrientes de agua. Novedad provincial.

Globularia alypum L.

Cehegín, próximo al Embalse de Argos (XH1126), $450 \mathrm{~m}, 1-\mathrm{IV}-1985, \mathrm{n} 2$ reg. 11480; Moratalla, hacia las Murtas (XHO336), 550 m, 5-V-1984, no reg. 
11359. Poco frecuente, en romerales sobre suelos poco profundos.

\section{Inula montana $\mathrm{L}$}

Moratalla, Rincón del Sastre (WH7029), 1200 m, 27-VI-1984, nํ reg.

12425. Escasa, en pedregales calizos. La cita de Gandoger (1.c.: 186) para Murcia (Calar del Mundo) corresponde a Albacete. Novedad provincial.

Ionopsidium prolongoi (Boiss.) Batt.

Moratalla, Sierra de Moratalla, Revolcadores (WH6413), $2000 \mathrm{~m}$, 7-VI-1981, no reg. 12432. Rara, en prados de anuales sobre suelos calizo-dolomíticos poco profundos. Novedad provincial.

Juniperus comunis L. subsp. hemisphaerica (J . \& C. Presl) Nyman

Moratalla, Sierra de Moratalla, Revolcadores (WH6412), $1900 \mathrm{~m}$, 1-XI-1984, no reg. 12486. Escasa, en suelos calizo-dolomiticos algo profundos. Novedad provincial.

Legousia hybrida (L.) Delarbre

Moratalla, Sierra de la Muela (WH9136), 900 m, 28-IV-1984, nํ reg.

12483. Escasa, en campos incultos. Novedad provincial.

Lepidium campestre (L.) R. Br.

Moratalla, Cortijo de la Pedregueruela (WH8828), 1100 m, 29-V-1982, no

reg. 12431. Poco frecuente, en márgenes de caminos. Novedad provincial.

Lepidium hirtum (L'.) subsp. hirtum

Moratalla, Arroyo Tercero (WH6826), 1100 m, 28-V-1983, no reg. 12430.

Poco frecuente, en campos incultos. Novedad provincial.

Malcomia ramosissima (Desf.) Thell

Moratalla, Cortijo Tercero (WH9529), 700 m, 21-V-1983, no reg. 12433.

Rara, en lugares incultos sobre suelos arenosos. Novedad provincial.

Pistorinia hispanica (L.) DC.

Moratalla, Cortijo Los García (WH8531), 1200 m, 27-VI-1984, no reg.

12482. Poco frecuente, en pedregales calizos. La cita de Gandoger (1.c.: 123) para Murcia (Sierra de Segura) debe ser referida a Albacete. Novedad provincial.

Polycarpon diphyllum Cav.

Calasparra, Sierra de la Puerta (XH0925), 500 m, 13-V-1984, no reg.

12497. Rara, en lugares ruderalizados. Novedad provincial.

Ptilostemon hispanicus (Lam.) W. Greuter

Caravaca, Cortijo de la Umbría (WH9424), 1000 m, 4-VII-1982, no reg.

12427. Poco frecuente, en suelos pedregoso calizos. Novedad provincial.

Salix x rubens Schrank

(S. alba L. X S. fragilis L.)

Moratalla, Arroyo Blanco (WH7024), 1100 m, 30V-1981, no reg. 11436.

Escasa en bordes de arroyo. Novedad provincial.

Saxifraga haenseleri Boiss. \& Reuter

Moratalla, Sierra de Moratalla, Revolcadores (WH6413), $1950 \mathrm{~m}$, 22-V-1982, no reg. 12485. Rara, en pedregales calizos. Novedad provincial.

Trifolium arvense L.

Moratalla, Cortijo Los García (WH8531), 1200 m, 27-VI-1984, no reg. 12492. Rara, en pastizales terofíticos sobre suelos arenosos. Novedad provincial. 
Veronica beccabunga L.

Moratalla, Sierra de Cantalar (WH7322), 1500 m, 30-V-1981, no reg. 12481. Escasa, en márgenes de corrientes de agua. Novedad provincial.

Vicia tenuifolia Roth. var. latifolia Lange In Willk. \& Lange Prodr. Fl. Hisp. 3:303 (1877).

Moratalla, Cortijo Los Asares (WH9327), $1100 \mathrm{~m}, 1-\mathrm{VI}-1985, \mathrm{n}$ ㅇ reg. 12499. Poco frecuente, en bordes de cultivos. Novedad provincial.

\title{
BIBL IOGRAF IA
}

ALCARAZ, F. \& MARTIN, M. -1984- Datos florísticos sobre la Sierra de Espuña (Murcia, España). Lazaroa, 6:259-261.

GANDOGER, M. -1917- Catalogue des plantes récoltées en Espagne et en Portugal pendant mes voyages de 1884 a 1892 . Paris.

SELMA, C. \& SOCORRO, O. -1985- Contribución al conocimiento de la flora del NW de Murcia. I. Anales de Biología (Secc. Biol. Veg. 1) 6:89-91. Univ. de Murcia.

\section{UNA NUEVA ASOCIACION CANTABRICA DE LA CLASE POLYGONO-POETEA ANNUAE: POO ANNUAE-SPERGULARIETUM SALINAE}

\author{
Mercedes HERRERA, Carlos AEDO \\ Tomás E. DIAZ \& José A. FERNANDEZ-PRIETO
}

RESUMEN: Se lleva a cabo una revisión de las comunidades pioneras y nitrófilas propias de biótopos intensamente pisoteados (Polygono-Poetea annuae) existentes en los territorios colinos y montanos de la cornisa Cantábrica (provincias Cántabro-Atlántica y Orocantábrica). Se reafirma la existencia en estas áreas de comunidades referibles a la Polygono-Matricarietum matricarioidis, Bryo-Saginetum procumbentis, Polygono-Coronopetum squamati, y, posiblemente, de la Crassulo tillaeae-Saginetum apetalae. Se describe una nueva asociación que se desarrolla en suelos con un cierto grado de salinidad en las áreas portuarias y sendas de los acantilados del litoral cantábrico: Poo annuae-Spergularietum salinae. 